\title{
Magnetic/Silica Nanocomposites as Dual-Mode Contrast Agents for Combined Magnetic Resonance Imaging and Ultrasonography
}

\author{
Maria Ada Malvindi, Antonio Greco, Francesco Conversano, Albert Figuerola, Maurizio Corti, \\ Marco Bonora, Alessandro Lascialfari, Houshang Amiri Doumari, Marco Moscardini, \\ Roberto Cingolani, Giuseppe Gigli, Sergio Casciaro, Teresa Pellegrino, and Andrea Ragusa*
}

A simple and efficient method for synthesizing a range of hybrid nanocomposites based on a core of silica nanospheres $(160,330$, and $660 \mathrm{~nm}$ in diameter) covered by an outer shell of superparamagnetic nanoparticles, either iron oxide or heterodimeric FePt-iron oxide nanocrystals, is presented. The magnetic and ultrasound characterization of the resulting nanocomposites shows that they have great potential as contrast agents for dual-mode imaging purposes, combining magnetic resonance imaging (MRI) and ultrasonography (US).

\section{Introduction}

Detection of diseases at an early stage of their development is a key issue in medicine. For instance, the earlier a cancer is diagnosed, the better are the chances of a successful treatment.

M. A. Malvindi, Prof. G. Gigli, Dr. T. Pellegrino, Dr. A. Ragusa

National Nanotechnology Laboratory of CNR-NANO

via per Arnesano km 5, 73100 Lecce, Italy

E-mail: andrea.ragusa@unile.it

M. A. Malvindi

Italian Institute of Technology

Center for Biomolecular Nanotechnology (CBN)

Via Barsanti 1, 73010 Arnesano, Lecce, Italy

A. Greco, Dr. F. Conversano, Dr. S. Casciaro

Consiglio Nazionale delle Ricerche - Istituto di Fisiologia Clinica (CNR-IFC)

c/o Campus Ecotekne, via per Monteroni, 73100 Lecce, Italy

Dr. A. Ragusa, Dr. A. Figuerola, Prof. R. Cingolani, T. Pellegrino

Italian Institute of Technology

via Morego 30, 16163 Genova, Italy

Prof. M. Corti, Dr. M. Bonora, Prof. A. Lascialfari, M. Moscardini

Dipartimento di Fisica "A. Volta"

INSTM and CNISM, Università degli Studi di Pavia

Via A. Bassi 6, 27100 Pavia, Italy

Prof. M. Corti, Prof. A. Lascialfar

S3 Istituto di Nanoscienze-CNR

Via Campi 213/A, 41125 Modena, Italy

Prof. A. Lascialfari, Dr. H. A. Doumari

Dipartimento di Scienze Biomolecolari applicate ai Biosistemi and INSTM Università degli Studi di Milano

Via Trentacoste 2, 20134 Milano, Italy

DOI: 10.1002/adfm.201100031
Many imaging techniques are currently available for identifying anatomical structures and functional activities inside the body, such as X-rays, magnetic resonance imaging (MRI), positron emission tomography (PET), single-photon emission tomography (SPECT), and ultrasonography (US). However, the above mentioned techniques are characterized by different image resolutions and information content. Hence, combination of multimodal data is often required in order to successfully diagnose a disease.

The employment of probes that enhance the image contrast allows higher resolutions than can be achieved otherwise. On the other hand, each different imaging probe has different pharmacodynamic properties, thus, the simultaneous visualization of the same site simply by summing the output of two or more different techniques is not usually feasible. In the last decades, great effort has been addressed toward the development of multimodal probes that can be detected by different techniques in real time. Several excellent reviews have been recently written on this topic. ${ }^{[1]}$ From a nanotechnological point of view, combined fluorescent-magnetic probes are probably the most investigated system, where the association of quantum dots (QDs) and superparamagnetic iron oxide nanoparticles allows for high visual sensitivity and spatial resolution. ${ }^{[2]}$ PET/MRI is another combined system that is rapidly developing because of the advantages of combined high soft-tissue contrast and functionality. ${ }^{[3]}$ From a medical point of view this might lead to improved diagnostic accuracy and simultaneous therapy. However, PET is a very expensive imaging technique that implies the use of highly ionizing radiation.

In this context, the development of a novel class of dualmode contrast agents, effectively detectable through both US and MRI, would bring significant improvements with respect to the currently adopted strategies for clinical management of several pathologies, especially tumors. ${ }^{[4]}$ In fact, apart from the complete absence of ionizing radiation and the strong general cost reduction for society, additional advantages are related to employing US for a first tumor localization and, where necessary, MRI could be exploited for a second-level investigation if more accurate high-resolution tumor segmentation is necessary, for instance, for the surgery pre-planning phase. Furthermore, 
US could be again used as a real-time guidance during surgical resection, supporting surgeons in appropriately defining the safety resection margins. All the mentioned imaging steps combine the benefits of both non-ionizing techniques (US and $\mathrm{MRI})$ requiring a single-contrast agent injection.

Recently, we demonstrated that silica nanoparticles (Si NPs) can be successfully exploited as ultrasound contrast agents at conventional diagnostic frequencies. ${ }^{[5]}$ The systematic study quantitatively investigated in vitro the ultrasound signal enhancement by varying both the dimension of the particles (from 160 to 330 and $660 \mathrm{~nm}$ in diameter) and the particle concentration in a range of clinical interest for tumor targeting purposes (from $2.5 \times 10^{10}$ to $1.5 \times 10^{13}$ particles $\mathrm{mL}^{-1}$ ). A linear relationship between particle concentration and mean amplitude of the backscattered ultrasound signal was observed for each tested dimension. Also, a strong power relationship between signal amplitude and particle diameter was found by analyzing samples with the same number of particles. On the other hand, the image brightness was found to be best enhanced by silica nanoparticles of $330 \mathrm{~nm}$ in diameter if the concentration in volume, that is the volume occupied by the nanoparticles at a fixed concentration, was considered. Compared to the other two dimensions (160 and $660 \mathrm{~nm}$ ), the 330-nm particles could also be advantageous for in vivo applications because of their potential ability of escaping the reticulo-endothelial system (RES) (whose action is more effective on larger particles) and, thus, avoiding to be trapped by intracellular openings in the normal vessel walls $(<50 \mathrm{~nm})$.

Starting from those results, we implemented a magnetic component onto the silica nanoparticles in order to create a bimodal US/MRI contrast agent. Either superparamagnetic iron oxide (IO) nanoparticles or dumbbell-like iron platinum-iron oxide (FePt-IO) nanocrystals were covalently linked to the outer surface of the silica nanoparticles, thus yielding a magnetic shell. IO NPs are well known and have already been extensively used as MRI contrast agents, ${ }^{[6]}$ whereas dimeric FePt-IO nanocrystals have been recently reported to yield even better image contrast compared to commercial $\mathrm{T}_{2}$ contrast agents, by carefully choosing the size of the FePt and the IO domains within the nanostructures. ${ }^{[7]}$ A combination of both units, the silica and the magnetic nanoparticles, yields multifunctional nanospheres and here we prove their use as dual-mode imaging probes for MRI and US techniques.

\section{Results and Discussion}

\subsection{Synthesis of the Bioconjugated Nanocrystals}

Silica nanoparticles were synthesized by a modified Stöber method. ${ }^{[8]}$ The reaction involved classical basic hydrolysis of tetraethylorthosilicate (TEOS) but it was accelerated by sonicating the mixture for $1 \mathrm{~h}$. Silica NPs with a diameter of 160,330 , and $660 \mathrm{~nm}$ were synthesized based on the positive response from previously reported ultrasonic attenuation data. ${ }^{[5]}$ The as-synthesized NPs have hydroxyl groups that cover their surface, which have to be replaced by amino groups for subsequent conjugation to the carboxy-functionalized magnetic nanocrystals (NCs). Successful functionalization with aminopropyltriethoxysilane (APS) yielded positively charged nanoparticles, as confirmed by the consistent change from negative to positive in the $\zeta$-potential values (Table 1 ).

The magnetic nanocrystals, both IO and FePt-IO NPs, were synthesized in organic solvents by high-temperature colloidal methods. ${ }^{[7,9]}$ Superparamagnetic IO NPs (15 nm in diameter) were prepared by a seeded-growth method as previously reported by Sun et al. ${ }^{[9]}$ This type of NPs are already well known and have been extensively reported in the literature as MRI contrast agents because of their magnetic behavior and their biocompatibility, which perfectly suit their use in clinical applications, also leading to their commercialization (e.g., Resovist, etc.) and to their use in clinical trials. ${ }^{[6 a, 6 e, 10]}$ Heterodimeric FePt-IO nanocrystals, with a FePt magnetic domain of $5 \mathrm{~nm}$ in diameter and an iron oxide domain of $12 \mathrm{~nm}$ in diameter, have also been tested as magnetic components. This type of nanocrystal has already proven to be an excellent material for its magnetic properties and its potential use as MRI contrast agent. ${ }^{[7]}$ Figuerola et al. have recently shown that the intimate contact between the FePt and the iron oxide domains yields nanostructures with magnetic properties different from those of the individual components, and that these NPs could perform even better as MRI contrast agents compared to the commercial contrast agent Endorem $@$ (Guerbet Group, Roissy, France). ${ }^{[7]}$

The as-synthesized NCs were prepared in organic solvents and transferred into aqueous medium by intercalating with poly(maleic anhydride-alt-1-octadecene) and adding a triamine, which yielded water-soluble NPs with an outer shell made of several carboxylic groups, as confirmed by the negative $\zeta$-potential values of -25 and $-30 \mathrm{mV}$ for the IO and FePt-IO NCs, respectively. ${ }^{[11]}$ Direct addition of the solution with anhydride-coated magnetic NCs to a suspension of APS-functionalized silica NPs led to the attachment of only a few NCs through formation of amide bonds between the amino-functionalized silica

Table 1. Comparison between transmission electron microscopy (TEM) and dynamic light scattering (DLS) measurements and corresponding $\zeta$-potential values in aqueous media.

\begin{tabular}{lccc}
\hline & TEM $[\mathrm{nm}]$ & DLS $[\mathrm{nm}]$ & $\zeta$-potential $[\mathrm{mV}]$ \\
\hline IO & 15 & 25 & -25 \\
FePt-IO & 17 & 25 & -30 \\
160Si-OH & 160 & 180 & -33 \\
160SI-NH 2 & 160 & 180 & +34 \\
160Si@IO & 190 & 240 & -19 \\
160Si@FePt-IO & 195 & 280 & -16 \\
330Si-OH & 330 & 400 & -40 \\
330SI-NH 2 & 330 & 400 & +40 \\
330Si@IO & 360 & 410 & -20 \\
330Si@FePt-IO & 365 & 420 & -16 \\
660Si-OH & 660 & 700 & -45 \\
660SI-NH & 660 & 700 & +45 \\
660Si@IO & 690 & 710 & +4 \\
660Si@FePt-IO & 695 & 720 & +4 \\
\hline
\end{tabular}


nanoparticles and the anhydride-functionalized nanocrystals (data not shown). The alternative electrostatic interaction between the negatively charged NCs and the positively charged Si NPs also led to assembled nanocomposites that were, however, quite unstable depending on the environmental conditions $(\mathrm{pH}$, ionic strength, etc., data not shown). The chosen way to saturate the surface of the silica NPs with magnetic NCs was by covalently joining the carboxy-functionalized NCs and the amino-functionalized silica NPs through standard carbodiimide chemistry. ${ }^{[12]}$

After removing the excess magnetic nanoparticles in solution and poorly covered nanocomposites, TEM analysis of the products showed densely packed magnetic NPs covering the shell of the silica nanospheres (Figure 1). The magnetic components appear darker in the TEM images because of the strong scattering of the electron beam compared to the amorphous silica. In the FePt-IO NCs it is even possible to distinguish the two magnetic domains, the IO domain exhibiting a lower image contrast compared to the FePt domain (inset in Figure 1g). Successful conjugation between the two types of NPs was also confirmed by the significant increase of the hydrodynamic diameter values of the silica nanoparticles, as determined by DLS measurements (Table 1).

Typical scanning electron microscopy (SEM) images are shown in Figure 2. No aggregation is observed even on a large scale and also with this technique it is possible to observe the magnetic NPs (both IO and FePt-IO NPs) as bright spots covering the silica nanospheres quite homogeneously.

Statistical image analysis of the electron microscopy images of the samples showed that $90 \pm 20$ magnetic nanoparticles covered the shell of the $160-\mathrm{nm}$ Si nanospheres. This value increased to $140 \pm 30$ in the case of the $330-\mathrm{nm} \mathrm{Si}$, and to $170 \pm 30$ for the $660-\mathrm{nm}$ Si. This trend is quite consistent with the loading of Fe content measured by elemental analysis for the different silica samples, showing an increase in its ratios of 1:1.4:1.6 for the 160:330:660 nanospheres, respectively.

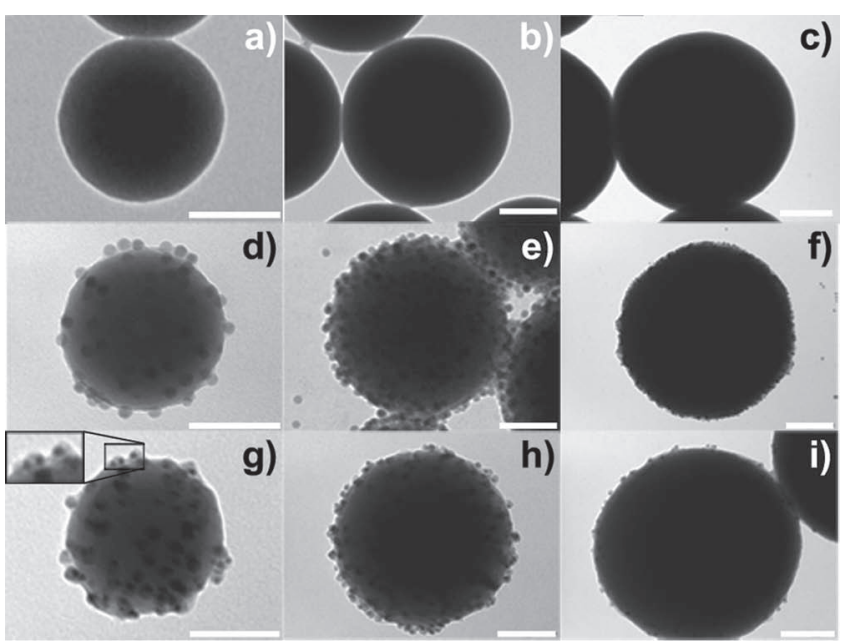

Figure 1. TEM images of $a-c)$ the starting Si NPs, $d-f$ ) the Si@IO NPs, and g-i) the Si@FePt-IO NPs. The first column represents the 160-nm NPs $(a, d, g)$, the second column shows the 330-nm NPs (b,e,h), and the third column shows the 660-nm NPs $(c, f, i)$. The scale bars are $100 \mathrm{~nm}$ $(a, b, d, e, g, h)$, and $200 \mathrm{~nm}(c, f, i)$.

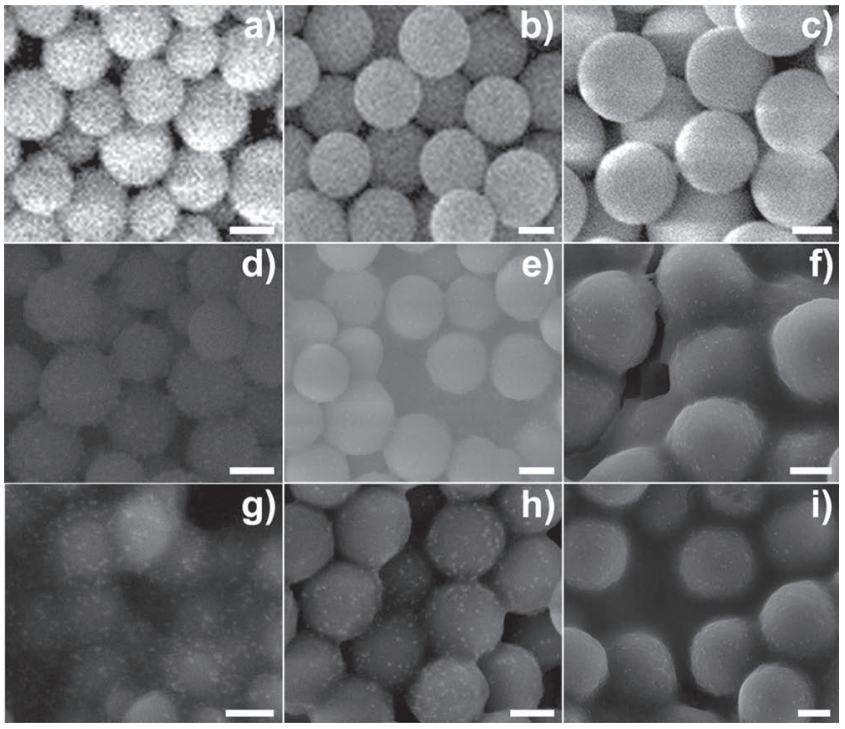

Figure 2. SEM images of $a-c)$ the starting Si NPs, $d-f$ ) the Si@IO NPs, and g-i) the Si@FePt-IO NPs. The first column shows the 160-nm NPs $(a, d, g)$, the second column the 330-nm NPs (b,e,h), and the third column the 660-nm NPs (c,f,i). The scale bars are $100 \mathrm{~nm}(\mathrm{a}, \mathrm{d}, \mathrm{g}), 200 \mathrm{~nm}(\mathrm{~b}, \mathrm{e}, \mathrm{h})$, and $300 \mathrm{~nm}(\mathrm{c}, \mathrm{f}, \mathrm{i})$.

\subsection{SQUID Magnetic Measurements}

Zero-field-cooling (ZFC) and field-cooling (FC) experimental results were collected on different samples at $H=150$ Oe (Figure 3 ) and the relevant parameters are listed in Table 2. In Figure 4 the hysteresis curves collected at $T=5 \mathrm{~K}$ are reported. In Figures 3 and 4 the typical behavior of superparamagnetic samples is evidenced. It should be noted that the reported quantity is the "effective" magnetization $M_{\text {eff }}$, in other words, the total magnetic moment divided by the iron concentration of each sample.

From Figure 3 it can easily be deduced that the blocking temperature, $T_{\mathrm{B}}$ (corresponding to the maximum in the ZFC curves), depends very slightly on the silica nanospheres diameter $d$, showing a trend to increase with $d$. The percentage variation of $T_{\mathrm{B}}$ with $d$ is, however, limited to about 7-8\% and it occurs only when comparing the $160 \mathrm{Si}$ to the $330 \mathrm{Si}$ samples. Thus, one can tentatively attribute this variation to slight differences in the magnetic core diameters of the samples. A more exotic explanation could involve a possible role of $\mathrm{Si}$ in increasing the total magnetic moment but this fact has no theoretical base in our current state of knowledge. Additionally, one can observe that, at the same silica diameter, $T_{\mathrm{B}}$ for the IO NPs assumes very slightly lower (some percent) values with respect to those of FePt-IO NPs. Below $T_{B}$, the spin freezes and the system enters the blocked regime with typical out-ofequilibrium behavior. ${ }^{[13]}$ Hysteresis loops display small coercive fields $H_{\mathrm{c}}$ (given in Table 2) and a residual magnetization $M_{\mathrm{r}}$, as shown in Figure 4. In both IO and FePt-IO cases, by increasing the diameter of the silica nanospheres the (small) coercive field increases as well (maximum of about 20\%). At fixed silica diameter, the corresponding $H_{\mathrm{c}}$ for the FePt-IO nanoparticles is slightly higher than the one for IO nanoparticles. The slightly 

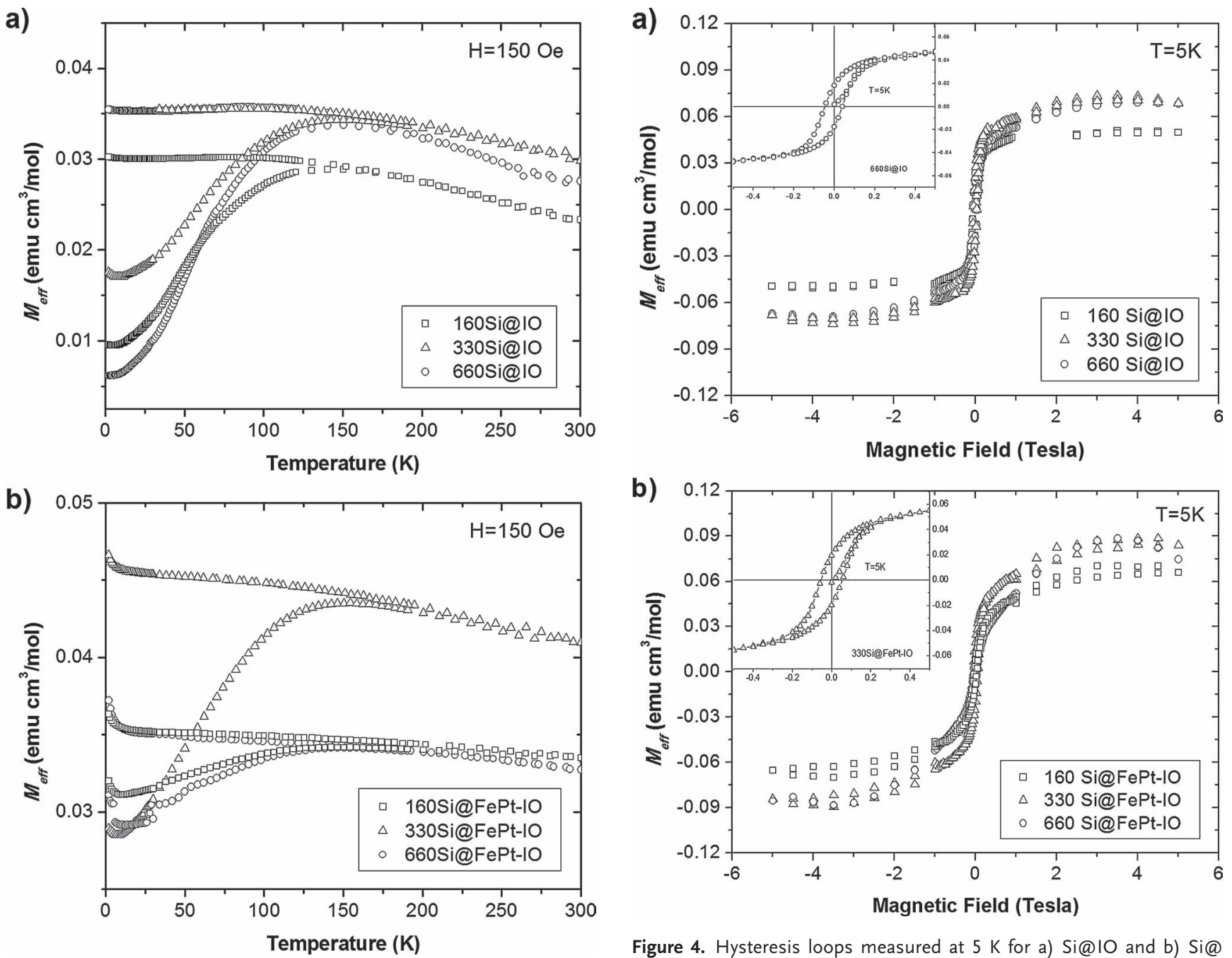

Figure 3. ZFC and FC magnetization measurements on a) Si@lO and b) Si@FePt-IO nanoparticles using the SQUID magnetometer.

higher values obtained for $H_{\mathrm{c}}$ in FePt-IO NPs, with respect to IO ones for the same total diameter, can be qualitatively justified by a slightly higher average diameter of the core containing magnetic ions $(5+12 \mathrm{~nm}$ for FePt-IO NPs, against $15 \mathrm{~nm}$ for IO), implying possibly a slightly higher magnetic moment.

\subsection{NMR Relaxometry and MRI}

${ }^{1} \mathrm{H}$ NMR relaxivity profiles were determined by measuring the longitudinal and the transverse nuclear relaxation times

Table 2. Blocking temperature, $T_{\mathrm{B}}$, and coercive field, $H_{\mathrm{C}}$, obtained by SQUID for all measured nanoparticles. The error for $T_{\mathrm{B}}$ is $\pm 4.0 \mathrm{~K}$ and the one for $H_{c}$ is \pm 20 Oe.

\begin{tabular}{|c|c|c|c|c|c|c|}
\hline & $\begin{array}{l}\text { 160Si } \\
@ \text { @IO }\end{array}$ & $\begin{array}{l}330 \mathrm{Si} \\
@ 10\end{array}$ & $\begin{array}{l}660 \mathrm{Si} \\
@ 10\end{array}$ & $\begin{array}{c}160 \mathrm{Si} \\
@ \mathrm{FePt}-\mathrm{IO}\end{array}$ & $\begin{array}{c}330 \mathrm{Si} \\
@ \mathrm{FePt}-\mathrm{IO}\end{array}$ & $\begin{array}{c}\text { 660Si } \\
@ \mathrm{FePt}-\mathrm{IO}\end{array}$ \\
\hline$T_{\mathrm{B}}[\mathrm{K}]$ & 140.4 & 150.4 & 150.4 & 142.4 & 154.4 & 154.3 \\
\hline$H_{\mathrm{C}}[\mathrm{Oe}]$ & 362.9 & 454.5 & 507.5 & 365.9 & 573.6 & 574.6 \\
\hline
\end{tabular}

Figure 4. Hysteresis loops measured at5 K for a) Si@IO and b) Si@ FePt-IO nanoparticles. The insets in (a) and (b) are enlarged views of the loops of660Si@IO and330Si@FePt-IO, respectively.

$T_{1}$ and $T_{2}$ at three RF frequencies: 12.5, 23, and $60 \mathrm{MHz}$. The measuring frequencies were chosen in order to collect data at magnetic fields near to the typical ones of the MRI images used in clinical studies and in research laboratories on animals and phantoms, namely $H=0.2,0.5$, and 1.5 Tesla. The nuclear relaxation times have been evaluated by using standard NMR radio frequency pulse excitation. In particular the transverse relaxation times $T_{2}$ were measured by using the Carr-Purcell-Meiboon-Gill (CPMG) pulse sequence to kill the spin diffusion effect in a non-homogeneous static magnetic field. ${ }^{[14]}$ The difference in $T_{1}$ and $T_{2}$ with respect to the values obtained at physiological temperature are within $10 \%$.

As it is well known, the contrast efficiency of the MRI contrast agents is determined by the relaxivities $r_{1,2}$ defined as: ${ }^{[15]}$

$r_{\mathrm{i}}=\left[\left(1 / T_{\mathrm{i}}\right)_{\text {meas }}-\left(1 / T_{\mathrm{i}}\right)_{\text {dia }}\right] / c \quad \mathrm{i}=1,2$

where $\left(1 / T_{\mathrm{i}}\right)_{\text {meas }}$ is the value measured on the sample with a concentration $c$ of the magnetic center (i.e., the iron content in IO and FePt-IO dimers), and $\left(1 / T_{\mathrm{i}}\right)_{\mathrm{dia}}$ is the nuclear relaxation rate 
a)

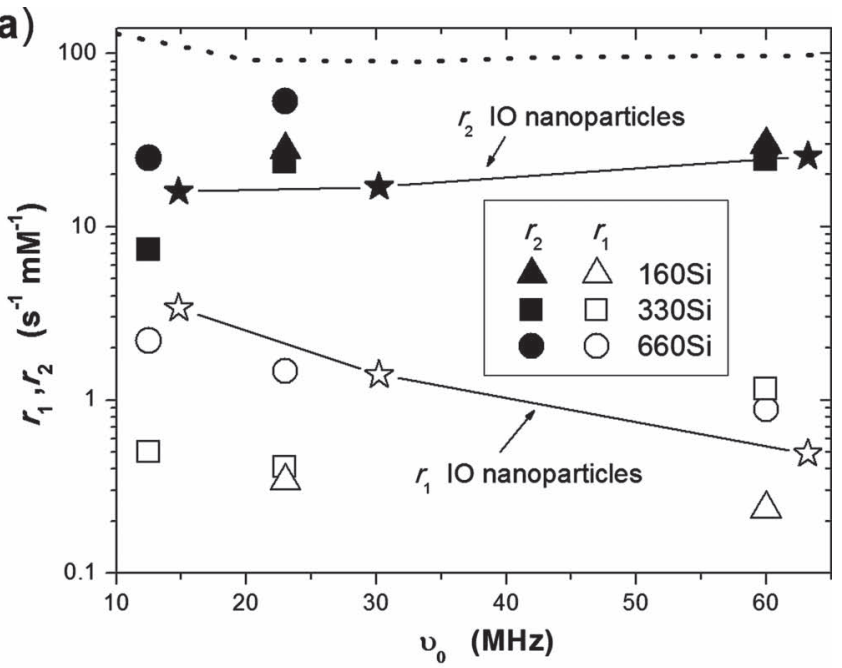

b)

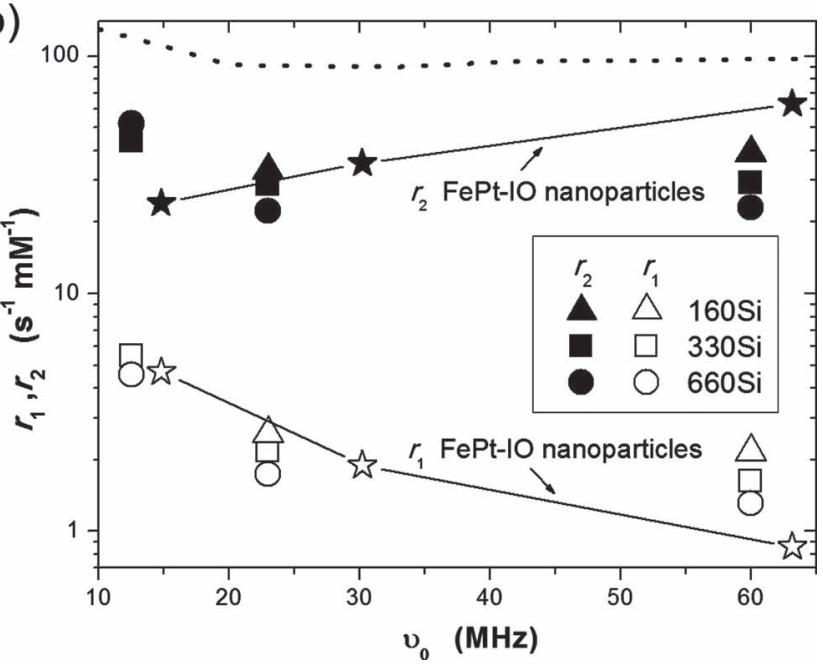

Figure 5. Longitudinal $r_{1}$ (open symbols) and transverse $r_{2}$ (full symbols) nuclear relaxivities of different nanospheres covered with a) IO or b) FePt-IO NPs. The $r_{1}$ and $r_{2}$ data are compared to that of pure IO or FePt-IO nanoparticles (stars). The $r_{2}$ data of the commercial compound

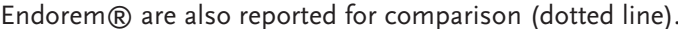

of the diamagnetic host solution. In our case, the different silica nanospheres $(160,330$, and $660 \mathrm{~nm}$ in diameter) both covered and not-covered by magnetic NPs were dispersed in agarose gel.

In Figure 5 the $r_{1}$ and $r_{2}$ values measured for the different silica host nanospheres covered by IO or FePt-IO nanoparticles are reported as a function of the measuring proton NMR frequency. ${ }^{[16]}$ For comparison, the relaxivities $r_{1}$ and $r_{2}$ of the pure $\mathrm{IO}$ and FePt-IO magnetic nanoparticles dispersed in agarose gel and $r_{2}$ of the commercial compound Endorem $₫$ (dotted line) are reported as well. [6c,7,17] As the ratio $r_{2} / r_{1}$ is greater than 2 , all the synthesized systems can be classified as good MRI negative contrast agents, in other words, $T_{2}$-relaxing systems. By looking at the results of Figure 5 it can be easily observed that they can be also usefully employed as good contrast agents for MRI imaging. In fact, although the $r_{2}$ values for the magnetic silica nanocomposites are lower than the commercial

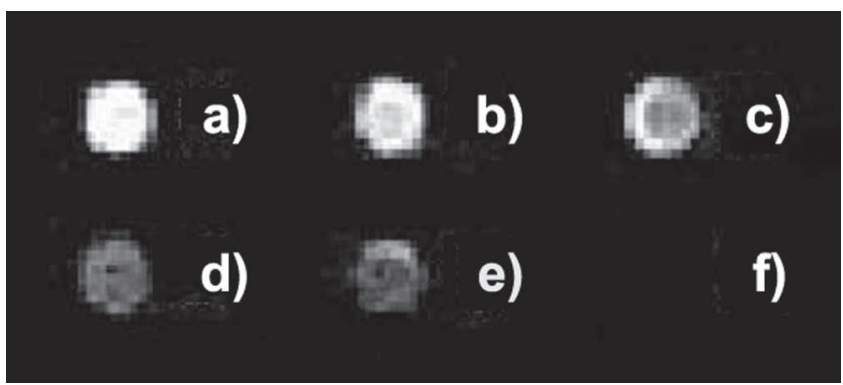

Figure 6. High-resolution gradient echo MRI at 0.2 Tesla on a phantom made with references and vials containing US samples characterized by different iron concentrations, all dispersed in agarose medium. The reference vials signals are: a) $330 \mathrm{Si}$; b) $660 \mathrm{Si}$. The US sample vials are: c)330Si@FePt-IO, [Fe]=0.1 mm; d) 660Si@FePt-IO, [Fe] = 0.2 mm; e)660Si@IO, $[\mathrm{Fe}]=0.3 \mathrm{~mm}$; f) 330Si@IO, [Fe]=1.0 mm. For iron concentrations greater than $0.1 \mathrm{~mm}$ the MRI signal is becoming increasingly darker; the $[\mathrm{Fe}]=1 \mathrm{~mm}$ sample is too dark to be visible.

Endorem $\AA$ ones, the values recorded are still significantly high to be visible in MRI images. This occurrence, together with a good US scattering ability, renders our systems good bimodal nanostructures for biomedical investigations.

To check the efficiency of our systems as negative MRI contrast agents, we collected MRI images of our Si-based NPs by means of a MRI tomograph (Esaote-Artoscan) operating at $H=$ $0.2 \mathrm{~T}$. The $T_{2}$-weighted image shown in Figure 6 was obtained using vials containing samples with silica nanospheres of different diameters covered by IO or FePt-IO nanoparticles, for comparison pure silica nanospheres of diameter $660 \mathrm{~nm}$ (660Si) and $330 \mathrm{~nm}(330 \mathrm{Si})$ are also shown.

Figure 6 shows that our samples show a good $T_{2}$-contrast, well evidenced by the comparison of the signal intensity of the pure nanospheres samples (660 Si and 330Si that gives a signal comparable to the one of water inside the human body) with respect to that of the magnetically functionalized samples: the last ones correctly act as negative contrast agents, giving darker images.

\subsection{Ultrasound Measurements}

Typical ultrasound images of the analyzed samples are reported in Figure 7. All the nanoparticle-containing phantoms produced a visible image enhancement (Figure 7a-i) with respect to the pure agarose gel, that was essentially transparent to ultrasound (Figure 7j). We can also notice that, among the three tested nanoparticle compositions, the pure silica nanospheres (Figure 7a-c) produced the highest image brightness for each considered diameter. The images obtained from Si@IO-containing phantoms were always characterized by the lowest brightness, but they also presented a clear trend for higher contrast levels with increasing particle size (Figure 7d-f). Finally, the Si@FePt-IO NPs (Figure 7g-i) showed image enhancements that are qualitatively analogous to those of silica NPs with a slightly less uniform brightness, especially for smaller diameter values.

These observations were quantitatively confirmed by the results of a frame-by-frame analysis of the ultrasound backscatter 

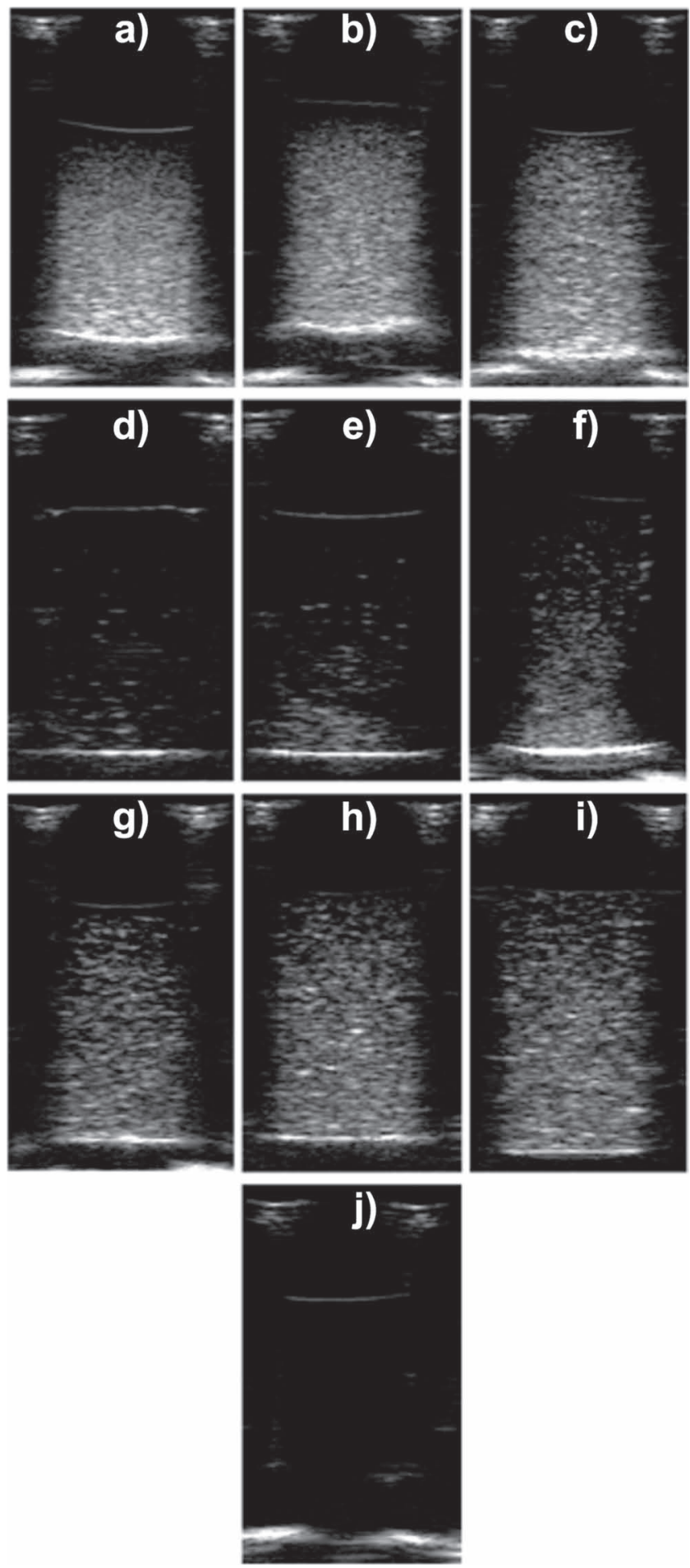

Figure 7. Typical ultrasound images of the analyzed agarose gel phantoms, containing nanoparticles of variable size and composition: a) 160-nm Si NPs, b) 330-nm Si NPs, c) 660-nm Si NPs, d) 160-nm Si@IO NPs, e) 330-nm Si@ IO NPs, f) 660-nm Si@IO NPs, g) 160-nm Si@FePt-IO NPs, h) 330-nm Si@ FePt-IO NPs, i) 660-nm Si@FePt-IO NPs, j) pure agarose gel.

amplitude (Figure 8). Silica NPs showed the strongest ultrasound backscatter power for each considered diameter, with a peak of maximum effectiveness corresponding to the $160-\mathrm{nm}$

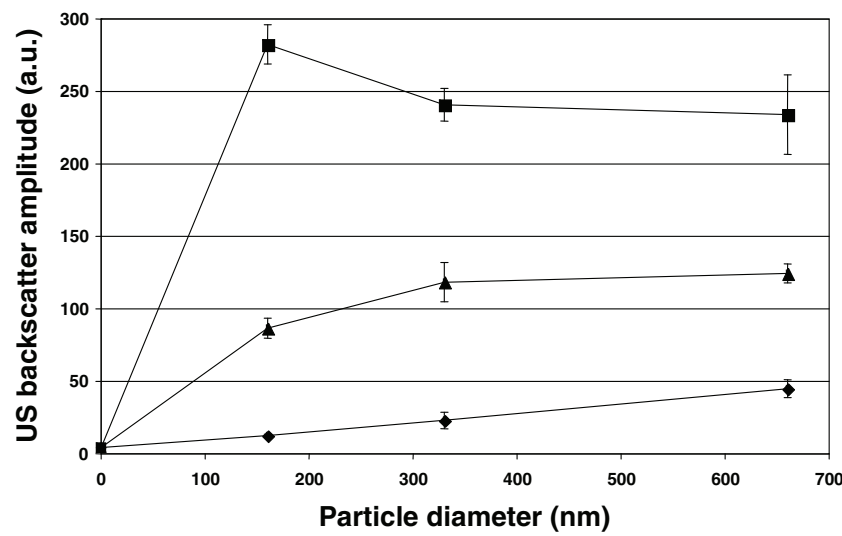

Figure 8. Mean amplitude of ultrasound backscatter within the considered region of interest (ROI) for Si NPs (squares), Si@FePt-IO NPs (triangles), and Si@IO NPs (diamonds). (Error bars represent standard deviations).

particles. On the other hand, the backscatter amplitudes of both nanocomposite systems presented lower values that increased with particle diameter. In addition, the curve of Si@FePt-IO NPs seems to level off in its final part, following the same trend as that of the silica NPs, with ultrasound signal amplitudes in the same order of magnitude, whereas the backscatter of Si@ IO NPs was one order of magnitude lower and showed a linearly increasing relationship with particle size.

The observed behavior of silica NPs is in good agreement with our recent findings on the combined effect of nanoparticle number and diameter on the related ultrasound image brightness, obtained with a $7.5 \mathrm{MHz}$ ultrasound frequency and particle volume concentrations in the range of $0.4-0.8 \% .{ }^{[5]}$ In the present paper, in fact, the employment of a lower volume concentration (about $0.2 \%$ ) and a higher ultrasound frequency $(10 \mathrm{MHz})$ can explain the shift of the peak in the ultrasound backscatter of silica particles from $330 \mathrm{~nm}$ to $160 \mathrm{~nm}$.

The lower measured backscatter of both nanocomposite systems can be related to two factors: first, the higher density of magnetic NPs with respect to silica reduces the actual nanoparticle concentration value; second, as we can see from the TEM images (Figure 1), the addition of the smaller magnetic NPs on the silica nanospheres alters the particle surface morphology in a significant and complex manner, and this is likely to affect the related ultrasound backscatter phenomena. The latter cause, in particular, can explain the absence of the peak in the backscatter curves of nanocomposite systems and the difference in amplitude range between the Si@FePt-IO NPs and Si@IO NPs.

\section{Conclusions}

A simple approach to generate water-soluble silica-based nanoparticles covered by a shell of magnetic particles was developed. Three diameters of the silica core were tested, 160-, 330-, and $660 \mathrm{~nm}$, whereas the magnetic components were comprised either of iron oxide nanoparticles (15 nm in diameter) or FePt-IO heterodimers (17 nm total length). The as-synthesized 
nanocomposites maintained the original characteristics of both types of nanoparticles, thus yielding a probe that could be used as contrast agent for combined MRI and US imaging. The reasonable contrast in MRI, together with a good US image contrast ability, makes our systems good bimodal nanostructures for biomedical investigations. Preliminary in vitro experiments, to test the biocompatibility of these systems, and in vivo trials on mice, to check their effective response to dual imaging, are now being carried out and the results will be presented in due course.

These composite nanoparticles can be easily functionalized with a wide variety of biomolecules and could be used for the targeted recognition and imaging of specific tissues/organs taking advantage of the properties of both techniques. Moreover, as silica NPs can be easily labeled with fluorescent dies, the generation of a ternary multimodal imaging system might be easily achieved, which would also show the spatial distribution of the nanosystem at a molecular level by confocal microscopy. ${ }^{[2 \mathrm{~b}, 2 \mathrm{~d}]}$ Last but not least, in addition to their imaging abilities, these nanocomposites could also be used for therapeutic purposes, such as hyperthermia treatment and magnetically guided delivery of drugs that could be loaded inside the silica core. ${ }^{[10,18]}$

\section{Experimental Section}

Chemicals: Iron pentacarbonyl $\left(\mathrm{Fe}(\mathrm{CO})_{5}, 98 \%\right)$, oleic acid $\left(\mathrm{C}_{17} \mathrm{H}_{33^{-}}\right.$ $\mathrm{CO}_{2} \mathrm{H}$ or OLAC, $\left.90 \%\right)$, oleyl amine $\left(\mathrm{C}_{17} \mathrm{H}_{33} \mathrm{NH}_{2}\right.$ or OLAM, $\left.70 \%\right)$, 1-octadecene $\left(\mathrm{C}_{18} \mathrm{H}_{36}\right.$ or ODE, $\left.90 \%\right)$, tetraethylorthosilicate (TEOS, \#86578), ammonium hydroxide $\left(\mathrm{NH}_{4} \mathrm{OH}, \# 320145\right)$, bis-(hexamethylene) triamine (\#14506), $\quad \mathrm{N}$-(3-dimethylaminopropyl)- $N$ '-ethylcarbodiimide hydrochloride (EDC, \#E7750) were purchased from Sigma-Aldrich. Platinum(II) acetylacetonate $\left(\mathrm{Pt}(\mathrm{acac})_{2}, 99 \%\right)$ was purchased from Strem. Agarose (D-1 low EEO, \#C1349) was purchased from Eppendorf. All solvents were purchased from J.T. Baker. OLAC, OLAM, and ODE were degassed under vacuum and stored under nitrogen in a glovebox at $-20^{\circ} \mathrm{C}$ before use. The syntheses of the magnetic nanoparticles (both $\mathrm{IO}$ and FePt-IO nanoparticles) were carried out under air-free conditions, using a standard Schlenk line setup. Ultrapure grade water with a conductivity of $18.2 \mathrm{M} \Omega \mathrm{cm}$ was used in all experiments. All chemicals were used as received.

Synthesis and Water-Solubilization of the Magnetic Nanocrystals: Iron oxide nanoparticles with a diameter of $15 \mathrm{~nm}$ and FePt-iron oxide heterodimer HNCs with diameters of 12 and $5 \mathrm{~nm}$ for the two cores, respectively, were synthesized according to already published procedures. ${ }^{[7]}$ Both magnetic nanocrystals were transferred from the organic phase into water by adopting a polymer-coating procedure using poly (maleic anhydride-alt-7-octadecene). ${ }^{[1]}$ The excess free polymer and other impurities were separated from the water-soluble nanocrystals through an ultracentrifugation step. ${ }^{[1 \mathrm{~b}]}$ A Beckman Coulter Optima LE-80K ultracentrifuge, equipped with a SW41 Ti rotor, was used at $33000 \mathrm{rpm}$. A continuous sucrose gradient (20-66\% sucrose density range) was used and the samples were centrifuged for $50 \mathrm{~min}$.

Synthesis of the Silica Nanoparticles: Silica NPs were synthesized through the Stöber method. ${ }^{[8]}$ In a typical procedure, $100 \mu \mathrm{L}$ of TEOS and $10 \mathrm{~mL}$ of $\mathrm{NH}_{4} \mathrm{OH}$ (28-30 wt.\%) were added to a vial containing $5 \mathrm{~mL}$ of EtOH $(95 \% \mathrm{v} / \mathrm{v})$ and the mixture was sonicated in a thermostatic bath at $25{ }^{\circ} \mathrm{C}$. A white turbid suspension was obtained after $1 \mathrm{~h}$. The $\mathrm{Si}$ NPs were then precipitated by centrifugation $(2000 \mathrm{rpm}, 20 \mathrm{~min}, T=$ $10{ }^{\circ} \mathrm{C}$ ), the supernatant was discarded, and the NPs were dispersed in a mixture of water/acetone $(1: 1)$. This purification step was repeated 5 times, after which the silica particles were collected and dried under vacuum at $-60^{\circ} \mathrm{C}$ for $5 \mathrm{~h}$. The above mentioned conditions yielded about $25 \mathrm{mg}$ of Si NPs with a diameter of $160 \mathrm{~nm}$ and a standard deviation below $5 \%$ (as determined by TEM statistical analysis).
The same synthetic procedure was followed to obtain Si NPs with different sizes by modifying the amounts of reagents involved in the reaction, namely, the concentration of TEOS, $\mathrm{NH}_{4} \mathrm{OH}$, and EtOH. In particular, Si NPs with a diameter of $330 \mathrm{~nm}$ were prepared by using $5 \mathrm{~mL}$ of EtOH, $120 \mu \mathrm{L}$ of TEOS, and $5 \mathrm{~mL}$ of $\mathrm{NH}_{4} \mathrm{OH}$; whereas Si NPs with a diameter of $660 \mathrm{~nm}$ were prepared by using $5 \mathrm{~mL}$ of $\mathrm{EtOH}$, $100 \mu \mathrm{L}$ of TEOS, and $1 \mathrm{~mL}$ of $\mathrm{NH}_{4} \mathrm{OH}$.

Synthesis of Amino-Functionalized Silica Nanoparticles: Silica NPs (30 mg) were dispersed in a freshly prepared solution of $1 \mathrm{~mm}$ acetic acid with $5 \%(\mathrm{v} / \mathrm{v})$ aminopropyltriethoxysilane (APS). The mixture was stirred for $1 \mathrm{~h}$ and then washed with acetone $(3 \times)$ and water $(3 \times)$.

Synthesis of the Hybrid Silica Nanoparticles: Water-soluble magnetic NPs $(25 \mu \mathrm{mol})$ were added to a solution of EDC $(50 \mu \mathrm{mol})$ in borate buffer $(250 \mu \mathrm{L})$ and the mixture was vortex-mixed for $30 \mathrm{~min}$. Amino-functionalized silica nanoparticles $(30 \mathrm{mg})$ in borate buffer $(250 \mu \mathrm{L})$ were added and the mixture was allowed to react for $12 \mathrm{~h}$. The nanoparticles were then purified through centrifugation $(3000 \mathrm{rpm}$, $10 \mathrm{~min}$ ) and magnetic separation in which the supernatant was discarded and the precipitated NPs were re-dispersed in borate buffer $(\mathrm{pH} 9)$. This purification step was repeated several times until the complete removal of free $\mathrm{IO}$ and FePt-IO nanoparticles in solution. The whole process of functionalization and purification was repeated if needed until the surface of the silica nanoparticles was fully covered by the magnetic nanoparticles, as observed by TEM analysis.

Dynamic Light Scattering (DLS) and Zeta-Potential Measurements: DLS and $\zeta$-potential measurements were performed with a Zetasizer Nano ZS90 (Malvern, USA) equipped with a $4.0 \mathrm{~mW}$ He-Ne laser operating at $633 \mathrm{~nm}$ and an Avalanche photodiode detector. Measurements were made at $25{ }^{\circ} \mathrm{C}$ on aqueous solutions $(\mathrm{pH} 7)$ of the particles. The values were determined using the Smoluchowsky approximation $\left(f\left(k_{\mathrm{a}}\right)=1.5\right)$, and were estimated as the average of twenty repeated measurements.

Transmission Electron Microscopy (TEM): Low-magnification TEM images were recorded on a JEOL JEM1011 microscope operating at an accelerating voltage of $100 \mathrm{kV}$. The samples for analysis were prepared by dropping a dilute solution of nanocrystals in water on carbon-coated copper grids (Formvar/Carbon $300 \mathrm{Mesh} \mathrm{Cu}$ ) and by allowing the solvent to evaporate. A statistical analysis was carried out on several wide-field, low-magnification TEM images, with the help of dedicated software (Image J 200). For each sample, 200 particles were counted at least.

Scanning Electron Microscopy (SEM): SEM characterization was performed with a RAITH 150 EBL instrument and the images were acquired at low accelerating voltages $(5 \mathrm{kV})$. The samples for analysis were prepared by dropping a suspension of the nanoparticles in water on silicon substrates and by letting the solvent evaporate.

Elemental Analysis: An inductively coupled plasma atomic emission spectrometer (ICP-AES) Varian Vista AX was used to measure the Fe content of all the purified samples. The samples were dissolved in concentrated $\mathrm{HCl} / \mathrm{HNO}_{3}(3: 1, \mathrm{v} / \mathrm{v})$ and directly analyzed after dilution in ultrapure grade water.

Magnetic Measurements: Magnetization measurements were performed on Si-based magnetic nanoparticles in agarose gel using a quantum design superconducting quantum interference device (SQUID) MPMS-XL7 magnetometer. The temperature dependence of the magnetization was studied in the temperature range of $2-300 \mathrm{~K}$, and zero-field-cooling (ZFC) and field-cooling (FC) curves were collected at an applied field $H=150$ Oe. To investigate the behavior of the magnetization as a function of the applied magnetic field, hysteresis experiments in the range of $-5 T \leq H \leq+5 T$ were performed at $T=5 \mathrm{~K}$. All the measurements were corrected by the diamagnetic contributions of $\mathrm{Si}$ and the sample holder.

NMR Relaxometry and MRI Measurements: The NMR measurements were performed at room temperature by using a Bruker-MLS 200 NMR-FT spectrometer and a Tecmag-Apollo FT-NMR spectrometer both equipped with an electromagnet. The nuclear relaxation times were evaluated at frequencies of $12.5,23$, and $60 \mathrm{MHz}$. In order to get rid of the spin-diffusion effect in a non-homogeneous magnetic field, we used the Carr-Purcell-Meiboon-Gill (CPMG) sequence for $T_{2}$ determination and the saturation-recovery sequence for $T_{1}$. The MRI images were 
obtained by means of an Artoscan Imager (Esaote SpA) operating at $H=0.2 \mathrm{~T}$. MRI images were recorded using a high-resolution gradient echo sequence with TR/TE/NEX $=900 \mathrm{~ms} / 8 \mathrm{~ms} / 4$, matrix $256 \times 192$, FOV $=$ $180 \times 180$, flip angle $=75^{\circ}$.

Ultrasound Measurements: Each prepared sample was imaged employing a modified version of an experimental set-up that we have recently developed for a similar purpose. ${ }^{[5]}$ We used a digital ultrasound machine (Megas GPX, Esaote Spa, Florence, Italy), equipped with a linear transducer (LA523, Esaote Spa), operating at $10 \mathrm{MHz}$, and linked via an optic fiber to a prototype platform for acquisition of unprocessed radiofrequency (RF) data (FEMMINA, ELEN Spa, Florence, Italy). The ultrasound probe was mounted on the motorized mechanism of an infusion pump (KDS 100, KD Scientific Inc., Holliston, MA) for automatic sample scanning. The analyzed Eppendorf was fixed at the bottom of a water-filled tank and the transducer, also partially immersed in the water, was positioned perpendicular to the agarose surface at such a distance that the transducer focus (set to $2 \mathrm{~cm}$ ) was located half-way through the nanoparticle-containing gel depth. For each scanned sample, 300 frames of RF data were acquired in its central part.

During the off-line analysis, the absolute value of high-pass filtered RF signals was identified as the ultrasound backscatter amplitude. For each acquired frame, a rectangular region of interest (ROI) composed of 25 ultrasound tracks with 360 points/track was positioned in the central zone of the nanoparticle-containing gel, so that the corresponding backscatter values were not influenced by any boundary effects. The mean backscatter amplitude of each frame was calculated as the average of the backscatter values of the 9000 points belonging to the ROI. The ultrasound backscatter amplitude associated to each sample was the average of 300 values resulting from the corresponding frame sequence.

\section{Acknowledgements}

The authors thank the European Commission for funding (Project Magnifyco, Contract NMP4-SL-2009-228622). These studies were partially supported by the grant $\mathrm{n}^{\circ}$ DM18604-Bando Laboratori-DD MIUR 14.5.2005 n.602/Ric/2005 of the Italian Ministry of Instruction and Research.

Received: January 2, 2011

Revised: March 1, 2011

Published online: May 9, 2011

[1] a) A. Louie, Chem. Rev. 2010, 110, 3146; b) R. Hao, R. Xing, Z. Xu, Y. Hou, S. Gao, S. Sun, Adv. Mater. 2010, 22, 2729; c) E.-K. Lima, J. Yang, C. P. N. Dinney, J.-S. Suh, Y.-M. Huh, S. Haam, Biomaterials 2010, 31, 9310

[2] a) S. A. Corr, Y. P. Rakovich, Y. K. Gun'ko, Nanoscale Res. Lett. 2008 3, 87; b) J. E. Lee, N. Lee, H. Kim, J. Kim, S. H. Choi, J. H. Kim, T. Kim, I. C. Song, S. P. Park, W. K. Moon, T. Hyeon, J. Am. Chem. Soc. 2010, 132, 552; c) W. C. Law, K. T. Yong, I. Roy, G. Xu, H. Ding, E. J. Bergey, H. Zeng, P. N. Prasad, J. Phys. Chem. C 2008, 112, 7972; d) J. H. Lee, Y. W. Jun, S. I. Yeon, J. S. Shin, J. Cheon, Angew. Chem. Int. Ed. 2006, 45, 8160; e) A. Quarta, R. Di Corato, L. Manna, A. Ragusa, T. Pellegrino, IEEE Trans. NanoBiosci. 2007, 6, 298; f) A. Quarta, R. Di Corato, L. Manna, S. Argentiere, R. Cingolani, G. Barbarella, T. Pellegrino, J. Am. Chem. Soc. 2008, 130, 10545.
[3] a) C. Glaus, R. Rossin, M. J. Welch, G. Bao, Bioconjug. Chem. 2010, 21, 715; b) M. S. Judenhofer, H. F. Wehrl, D. F. Newport, C. Catana, S. B. Siegel, M. Becker, A. Thielscher, M. Kneilling, M. P. Lichy, M. Eichner, K. Klingel, G. Reischl, S. Widmaier, M. Rocken, R. E. Nutt, H. J. Machulla, K. Uludag, S. R. Cherry, C. D. Claussen, B. J. Pichler, Nat. Med. 2008, 14, 459; c) T. Higuchi, M. Anton, K. Dumler, S. Seidl, J. Pelisek, A. Saraste, A. Welling, F. Hofmann, R. A. J. Oostendorp, B. Gansbacher, S. G. Nekolla, F. M. Bengel, R. M. Botnar, M. Schwaiger, J. Nucl. Med. 2009, 50, 1088.

[4] a) L. Curiel, R. Chopra, K. Hynyne, IEEE Trans. Med. Imaging 2007, 26, 1740; b) E. Pisani, N. Tsapis, B. Galaz, M. Santin, R. Berti, N. Taulier, E. Kurtisovski, O. Lucidarme, M. Ourevitch, B. T. Doan, J. C. Beloeil, B. Gillet, W. Urbach, S. L. Bridal, E. Fattal, Adv. Funct. Mater. 2008, 18, 2963; c) R. Díaz-López, N. Tsapis, E. Fattal, Pharm. Res. 2010, 27, 1.

[5] S. Casciaro, F. Conversano, A. Ragusa, M. A. Malvindi, R. Franchini, A. Greco, T. Pellegrino, G. Gigli, Invest. Radiol. 2010, 45, 715.

[6] a) H. B. Na, I. C. Song, T. Hyeon, Adv. Mater. 2009, 21, 2133; b) E. Taboada, R. Solanas, E. Rodriguez, R. Weissleder, A. Roig, Adv. Funct. Mater. 2009, 19, 2319; c) M. F. Casula, P. Floris, C. Innocenti, A. Lascialfari, M. Marinone, M. Corti, R. A. Sperling, W. J. Parak, C. Sangregorio, Chem. Mater. 2010, 22, 1739; d) O. Veiseha, J. W. Gunna, M. Zhang, Adv. Drug Delivery Rev. 2010, 62, 284; e) A. Figuerola, R. Di Corato, L. Manna, T. Pellegrino, Pharm. Res. 2010, 62, 126

[7] A. Figuerola, A. Fiore, R. Di Corato, A. Falqui, C. Giannini, E. Micotti, A. Lascialfari, M. Corti, R. Cingolani, T. Pellegrino, P. D. Cozzoli, L. Manna, J. Am. Chem. Soc. 2008, 130, 1477.

[8] W. Stöber, A. Fink, E. Bohn, J. Colloid Interface Sci. 1968, 26, 62.

[9] S. Sun, H. Zeng, J. Am. Chem. Soc. 2002, 124, 8204.

[10] J. Kim, Y. Piao, T. Hyeon, Chem. Soc. Rev. 2009, 38, 372.

[11] a) T. Pellegrino, L. Manna, S. Kudera, T. Liedl, D. Koktysh, A. L. Rogach, S. Keller, J. Radler, G. Natile, W. J. Parak, Nano Lett. 2004, 4, 703; b) R. Di Corato, A. Quarta, P. Piacenza, A. Ragusa, A. Figuerola, R. Buonsanti, R. Cingolani, L. Manna, T. Pellegrino, J. Mater. Chem. 2008, 18, 1991.

[12] G.-T. Hermanson, in Bioconjugate Techniques. Modification of Nucleic acids and Oligonucleotides (Ed.: G.-T. Hermanson), Academic Press, San Diego, CA, 1996, p. 77.

[13] a) S. Sun, Adv. Mater. 2006, 18, 393; b) U. Jeong, X. Teng, Y. Wang, H. Yang, Y. Xia, Adv. Mater. 2007, 19, 33.

[14] A. Lascialfari, M. Corti, in NMR-MRI, $\mu S R$ and Mossbauer Spectroscopies in Molecular Magnets (Eds.: P. Carretta, A. Lascialfari), Springer-Verlag, Milano, Italy, 2007, p. 89.

[15] a) S. Laurent, L. Vander Elst, A. Roch, R. N. Muller, in NMR-MRI, $\mu S R$ and Mossbauer Spectroscopies in Molecular Magnets (Eds.: P. Carretta, A. Lascialfari), Springer-Verlag, Milano, Italy, 2007, p. 71; b) R. N. Muller, Encyclopedia of Nuclear Magnetic Resonance, Wiley, New York, NJ, 1996.

[16] a) M. Corti, A. Lascialfari, M. Marinone, A. Masotti, E. Micotti, F. Orsini, G. Ortaggi, G. Poletti, C. Innocenti, C. Sangregorio, J. Magn. Magn. Mater. 2008, 320, E316; b) M. Corti, A. Lascialfari, E. Micotti, A. Castellano, M. Donativi, A. Quarta, P. D. Cozzoli, L. Manna, T. Pellegrino, C. Sangregorio, J. Magn. Magn. Mater. 2008, 320, E320.

[17] M. C. Franchini, G. Baldi, D. Bonacchi, D. Gentili, G. Giudetti, A. Lascialfari, M. Corti, P. Marmorato, J. Ponti, E. Micotti, U. Guerrini, L. Sironi, P. Gelosa, C. Ravagli, A. Ricci, Small 2010, 366.

[18] F. Gazeau, M. Lévy, C. Wilhelm, Nanomedicine 2008, 3, 831. 